\title{
Prevention Science Supplemental Issue Commentary Promoting Healthy Sexual Practices: What We Have Learned from 100 Years of Work
}

\author{
Deborah M. Capaldi
}

Published online: 7 September 2013

(C) Society for Prevention Research 2013

There are strong theoretical and empirical grounds for predicting that effective family focused interventions to prevent conduct problems and substance use should also prevent sexual risk behaviors. Spoth et al. (2013) considered it a "striking gap" that no studies could be found that examined the impact of such universal interventions on young-adult sexual risk behavior as mediated by substance use in adolescence. Yet, it is not so surprising when it is considered what must be accomplished in order to address this question. First, an effective prevention program must be designed and tested, and then many years of dedication to retaining participants and collecting further assessment waves for examining longer term outcomes are required of the investigators - as well as a tremendous sustained funding investment usually by federal agencies, in this case, predominantly NIDA. In fact, the combined studies reported here represent over 100 study years of work all told from planning to publication. From this point of view, it is amazing that six such studies can be presented in this issue!

There are a number of complex issues involved in examining prevention questions across young life spans, including heterogeneity in developmental pathways and how this relates to later outcomes (e.g., Conduct Problems Prevention Research Group 2013; Kellam et al. 2013), mediators such as positive family environments (e.g., Caruthers et al. 2013), and moderators, particularly including gender and ethnicity (e.g., Hill et al. 2013). Indeed, since many of the studies presented here began, statistical approaches have changed dramatically with the development of a variety of longitudinal methods to address critical key questions - including timing of developmental events, particularly, survival analyses (Hill et al. 2013); differential behavioral patterns and growth related to response to the intervention - including approaches using latent class analysis

D. M. Capaldi $(\bowtie)$

Oregon Social Learning Center, Eugene, OR, USA

e-mail: deborahc@oslc.org
(Conduct Problems Prevention Research Group 2013) and general growth mixture modeling (Kellam et al. 2013); structural equation and growth modeling examining direct and mediated pathways or indirect effects (Caruthers et al. 2013; Spoth et al. 2013); and examination of the practical significance of the effects on the outcomes by examining the relative reduction rates (in percentages) in health-risking sexual behaviors ( Spoth et al. 2013). Note that the Skinner et al. (2013) study was limited in the analytic approaches that could be taken by having just two waves of data. This issue showcases such methods due to the differing approaches the investigators took, related to their study questions, designs, and available data.

It is well established that there are associations of both conduct problems (including delinquency) and substance use with sexual risk behaviors in adolescence. A strong individual risk factor is poor inhibitory control and impulsivity. Whereas there is much evidence that other factors affect problem behavior involvement (e.g., deviant peer associations), poor inhibitory control has been established as a particularly strong mechanism (Gottfredson and Hirschi 1990).

A distinct difference between most types of delinquency and substance use and sexual activity is that sexual activity is a normal and important part of adult life, and some degree of substance use is also normative in most cultures. Both of these are intimately related to pleasure seeking. Whereas many individuals do not have the desire or impulse to commit a crime, most do have the desire to engage in substance use and sexual activity. It is not surprising then that there is an independent association of substance use and sexual activity in adolescence, even accounting for conduct problems. In fact, we found the association to be so strong (Capaldi et al. 1996) that adolescents showed distinctly different timing of increases in substance use by age of onset of intercourse such that the pace of substance use accelerated around the time of onset. There was very early engagement in these adult-like behaviors for some youth, and likely also combined substance 
use and sexual activity on social occasions. Substance use is strongly associated with lifetime sexual risk behavior through ages 22-23 years (Capaldi et al. 2002), and Spoth et al. (2013) found that just over one half of the young adults in their study reported having combined alcohol or drug use with sex.

One of the most successful early childhood interventions (in grade 1) presented here with regard to later sexual risk outcomes was the Good Behavior Game (Kellam et al. 2013), which showed effects assessed at ages 19-21 years for the highest-risk boys. This classroom-wide program taught inhibition of rule-breaking behavior, using a schedule for the game (from predictable to less predictable as the children progressed) that likely maximized the generalizability of the inhibition, particularly within the school setting. Note that rule-breaking behavior is the defining feature of conduct problems. It seems likely that much of the effectiveness of this program is in checking poorly controlled behaviors in children having the most difficulty in this area, and possibly shifting them onto a more normative downward trajectory (Gilliom and Shaw 2004). This then leads to other benefits, such as acceptance by prosocial peers, improved school performance, and possibly, child behavior that is easier for the parents to manage. It is certainly notable that this intervention had such strong long-term effects on substance use and sexual risk behavior without any intervention with the parents.

A second study to find particularly strong effects was that of Spoth et al. (2013). The study also involved a universal intervention but was focused on the families of sixth graders, and involved the parents and children in group sessions focused on skill building. Effects were found for reductions in substance use and to lower sexual transmitted infections (STIs) assessed at age 21 years, and mediational effects via lower growth in substance use were significant. Just prior to their children's adolescence, parents may be very receptive to working on the skills (e.g., constructive communication, problem solving, monitoring) needed to prevent children from becoming involved in substance use and sexual risk behaviors. Parental monitoring is associated with lower levels of sexual risk behaviors and substance use in adolescence (Lac and Crano 2009); thus, this may be a particularly effective approach at this age. Note also that, whereas the Kellam et al. (2013) sample was predominantly urban African American, the Spoth et al. (2013) children were predominantly White and rural. This is certainly encouraging in that salutary prevention effects of universal interventions were found for varied populations.

The Hill et al. (2013) study involved a comprehensive intervention involving the schools, children, and parents in grades 1 through 6, which was effective in preventing STIs through age 30 years (the longest follow-up period of all the studies), with the biggest effect being for African American participants - the ethnic group at highest risk of an STI. Here, intervention in grades 5 and 6 only were not effective. Tests of replications are thus needed to see whether a particular approach tends to work better with one population rather than another. The findings of these universal intervention studies suggest that some combination, such as a first-grade classroom intervention and a later sixth-grade family intervention may be particularly effective.

Three of the studies presented in this issue took on the challenging task of delivering interventions beyond universal, at the selective or tiered levels. In Fast Track (Conduct Problems Prevention Research Group 2013), children were screened in kindergarten, but the intervention didn't begin until grade 1 (and continued thru grade 10). Such children often come from homes with multiple and often severe problems (e.g., parental psychopathology and heavy substance use), are likely at genetic risk, and also have likely experienced poor parenting such as neglect and harsh discipline. Intervention effects were not found to the timing of first sexual intercourse, pregnancy, or STI contraction through grade 11 (ages 16-17 years). It is possible that significant prevention effects may be found at older ages, as in the prior studies discussed. The Caruthers et al. (2013) study involved a multilevel intervention with a brief motivational Family CheckUp followed by more intensive family based treatment where this was indicated for adolescents (grade 6) and their families. Whereas the intervention did not have direct effects on sexual risk behavior at age 22 years, indirect effects were found related to improvement in family relationship quality. Finally, the Skinner et al. (2013) study involved a family focused treatment for parents (with children aged 3-14 years) involved in methadone treatment and one follow-up conducted approximately 13 years later. The intervention was not found to affect the sexual risk behavior outcomes.

Given that the interventions showing stronger effects were, in general, universal in design, it is tempting to conclude that the effects in those studies were more likely for the moderately at-risk rather than the higher-risk youth. However, the Spoth et al. (2013) study had substantial effects on the outcomes, and the Kellam et al. (2013) study showed effects for the highestrisk boys. The GBG intervention may be particularly influential for high-risk children when delivered at age 6 to 7 years, and has the advantage that all children are included.

One difficult issue that was not addressed in these studies is the role of child sexual abuse in later sexual risk behaviors. A disturbingly large number of children and adolescents, particularly in high-risk populations, are coerced into sexual acts, which puts them at risk for ongoing sexual activity and STIs (Jones et al. 2010). This is a very difficult issue to tackle in research, particularly in a community study where school and family participation is severely compromised if such issues are assessed and reported to the police. If sexual abuse was assessed within prevention programs (at least when these are in dissemination rather than initial testing phase), it is possible that such children at high risk for STIs could be referred for more intensive prevention or treatment programs that could help them avoid problematic health outcomes. 
In conclusion, the studies presented here make exemplary use of prior preventive interventions to address the issue of promotion of long-term health by prevention of sexual risk behaviors. The findings of these studies provide very encouraging evidence that prevention of conduct problems and substance use using a variety of approaches and with differing populations can have multiple benefits stretching into adulthood. In particular, findings reinforce the fact that universal early interventions addressing root causes of such behaviors can be one of the most powerful and cost-effective approaches, considering the savings related to public health and crime avoidance that accumulate over time.

\section{References}

Conduct Problems Prevention Research Group (2013). Trajectories of risk for early sexual activity and early substance use in the fast track prevention program: Prevention Science. In press.

Capaldi, D. M., Crosby, L., \& Stoolmiller, M. (1996). Predicting the timing of first sexual intercourse for at-risk adolescent males. Child Development, 67, 344-359.

Capaldi, D. M., Stoolmiller, M., Clark, S., \& Owen, L. D. (2002). Heterosexual risk behaviors in at-risk young men from early adolescence to young adulthood: Prevalence, prediction, and association with STD contraction. Developmental Psychology, 38, 394-406.

Caruthers, A. S., Ryzin, M. J. V., \& Dishion, T. J. (2013). Preventing highrisk sexual behavior in early adulthood with family interventions in adolescence: Outcomes and developmental processes: Prevention Science. In press.

Gilliom, M., \& Shaw, D. S. (2004). Codevelopment of externalizing and internalizing problems in early childhood. Development and Psychopathology, 16, 313-333.

Gottfredson, M. R., \& Hirschi, T. (1990). A general theory of crime. Stanford: Stanford University.

Hill, K. G., Bailey, J. A., Hawkins, J. D., Catalano, R. F., Kosterman, R., Oesterle, S., Abbott, R. D. (2013). The onset of STI diagnosis through age 30: Results from the Seattle social development project intervention: Prevention Science. In press.

Jones, D. J., Runyan, D. K., Lewis, T., Litrownik, A. J., Black, M. M., Wiley, T., \& Nagin, D. S. (2010). Trajectories of childhood sexual abuse and early adolescent HIV/AIDS risk behaviors: The role of other maltreatment, witnessed violence, and child gender. Journal of Clinical Child and Adolescent Psychology, 39, 667-680.

Kellam, S. G., Wang, W., Mackenzie, A. C. L., Brown, C. H., Ompad, D. C., Or, F.,Windham, A. (2013). The impact of the good behavior game, a universal classroom-based prevention intervention in first and second grades, on high-risk sexual behaviors and drug abuse and dependence disorders into young adulthood. Prevention Science. In Press.

Lac, A., \& Crano, W. D. (2009). Monitoring matters: Meta-analytic review reveals the reliable linkage of parental monitoring with adolescent marijuana use. Perspectives on Psychological Science, 4, 578-586.

Skinner, M. L., Fleming, C. B., Haggerty, K. P., Catalano, R. F. (2013). Sex risk behavior among adolescent and young adult children of opiate addicts: Outcomes from the focus on families prevention trial and an examination of childhood and concurrent predictors of sex risk behavior: Prevention Science. In press.

Spoth, R., Clair, S., Trudeau, L. (2013). Universal family-focused intervention with young adolescents: Effects on health-risking sexual behaviors and STDs among young adults: Prevention Science. In press. 